\section{Time for More Inclusive Cancer Trials}

Piet Dirix, MD, PhD; Lynda Wyld, MD, PhD; Shani Paluch-Shimon, MBBS, MSc; and Philip Poortmans, MD, PhD

I $\mathrm{n}$ this issue of JNCCN, Abi Jaoude et al ${ }^{1}$ convincingly demonstrate that recruitment in randomized oncologic clinical trials is typically limited to patients with good performance status (PS). Indeed, in their review of 600 trials, the vast majority (96.3\%) of included patients had excellent PS (ECOG PS score 0 or 1 ). Certainly this is not consistent with daily clinical reality and, together with previously well-described age, sex, and race biases that are inherent in clinical trials, could severely limit the generalizability of trial results to routine clinical practice. ${ }^{2}$ Their thought-provoking analysis should encourage not only researchers and clinicians but also regulatory bodies, funders, and patient advocates to reflect critically about potentially serious biases in the processes to evaluate, approve, and reimburse new cancer treatments.

\section{General Age and Fitness Bias in Trial Recruitment}

The randomized clinical trial represents the gold standard evidence base for adoption of new clinical treatments. However, the data generated are only as valid as the methods used to obtain them. Recruitment bias is a major issue, with particular problems recruiting patients who are less healthy, whether due to age, comorbidities, or functional status. In many trials, the bias is inherent in the basic trial design, with preset age or PS cutoffs that result in a trial cohort not representative of the real-world population in which the treatment may be used after regulatory approval (Figure 1).

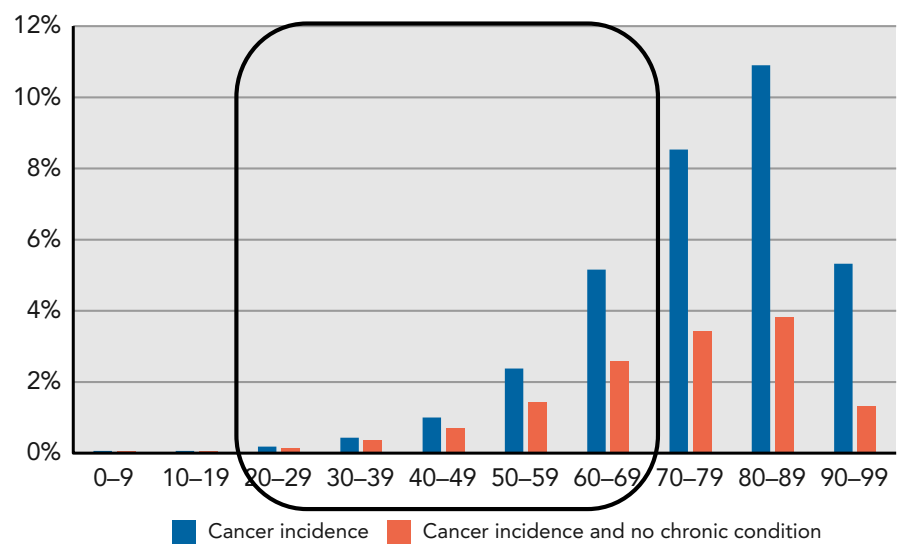

Figure 1. Patient eligibility based on age and PS. The blue bars display the age distribution for a total cancer lifetime incidence of $34.1 \%$. The orange bars indicate the proportion of patients with cancer who do not have another chronic health condition (and thereby assumed to have PS 0-1). The black box includes patients who are typically eligible for clinical trials, showing that only $5.2 \% / 34.1 \%=15.3 \%$ of all patients with cancer are expected to be in the acceptable age and PS range.

Abbreviation: PS, performance status.

See page 1322 for related article.

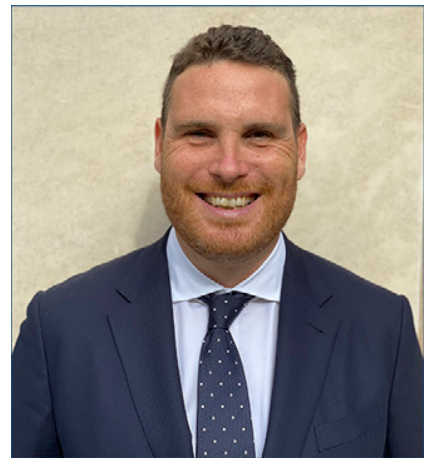

PIET DIRIX, MD, PhD

Piet Dirix, MD, PhD, works as a radiation oncologist in the Iridium Network in Antwerp (Belgium), where his focus is on pelvic genitourinary cancers. He is also an associate professor at Antwerp University and his main research interest is in the use of innovative imaging and stereotactic radiotherapy.

doi: $10.6004 /$ jnccn.2020.7652

The ideas and viewpoints expressed in this commentary are those of the author and do not necessarily represent any policy, position, or program of NCCN 


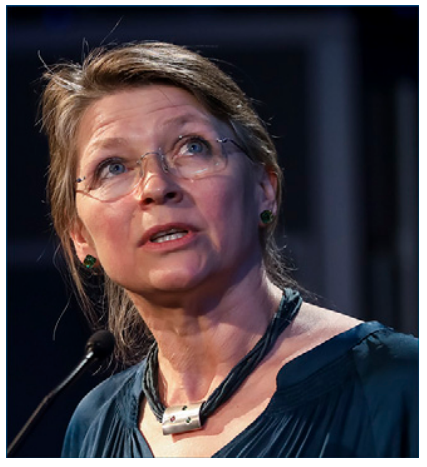

LYNDA WYLD, MD, PhD

Lynda Wyld, MD, PhD, is from the Department of Surgical Oncology, University of Sheffield, United Kingdom.
Of course, trial teams can have valid reasons why they may want to avoid less-fit patients. First, these patients are more likely to die of causes other than the disease under study, thus diluting the benefit of the trial intervention, and thereby reducing the power of the study or increasing the required sample size (and therefore costs). Second, adverse effects and treatment- or disease-related deaths may be more likely in patients with preexisting health issues, making the trial intervention clinically less beneficial and health-economically less attractive. Third, less-fit patients are more likely to be taking other medications, increasing the risk of drug interactions. Fourth, recruitment in older and less-fit patients is relatively more difficult due to both clinician and patient reticence about participating. ${ }^{3}$ Therefore, even if inclusion criteria are broad, differential recruitment may continue to occur. This is clearly apparent in the current analysis, in which even among trials that allowed patients with an ECOG $\geq 2$, only $8.1 \%$ of enrolled patients had a poor PS. ${ }^{1}$ Finally, trial compliance may be more challenging in less-fit patients, with higher rates of drop-outs and loss to follow-up.

As the age of the global population increases and more people are living with other chronic health problems, ensuring that trial data are representative of the realworld targeted population becomes increasingly important. ${ }^{4}$ Limiting trial recruitment to younger and fitter patients may result in invalid assumptions about benefits in the real-world population. This is especially the case for cancer trials, because many patients with cancer are older and less fit. ${ }^{5}$ Straightforward extrapolation of trial findings obtained from a selected population to patientsat-large may be dangerous, with increased risks of adverse effects. Conversely, treatments may not be offered to patients outside the inclusion criteria, which might potentially disadvantage large groups of patients that were unfairly not represented in the trials. It is therefore important that trial eligibility criteria closely reflect the likely real-world target population or, if not, adequate justification should be given. Also important, study authors must fully report differential recruitment and retention (and reasons) to inform readers and regulators about what clinical practice implications can be drawn from the data.

\section{Specific Problems With PS Scales}

Interestingly, both the Karnofsky and the ECOG PS scales were initially introduced to evaluate therapeutic efficacy and patient reaction to cancer drugs. In the 1970s, they transitioned to clinical trial eligibility and stratification with the intent to reduce variability and bias. Since then, PS as a fitness measure and eligibility criterion for inclusion in oncology trials remains widespread. However, although PS is validated and has some ability to predict mortality, it is far from ideal. In fact, several specific problems can be noted.

First, these scales are clinician-assessed and therefore highly subjective. Indeed, the interobserver variability in PS assessment among healthcare professionals is substantial. ${ }^{6}$ This is not surprising, because it depends on a "snapshot" interpretation of a patient's well-being. For instance, if an athlete in otherwise perfect health has a foot injury and is not allowed to put pressure on their foot, their ECOG PS should be scored as 2 if not 3 . Surely, many of us would, not unreasonably, score this patient as ECOG 0 or 1 . This inevitable ambiguity results in poor overall reliability of PS scoring. Conversely, it is also imaginable, if not likely, that the subjective scoring of PS leads to a score that more likely fits the trial requirements.

Second, there is poor concordance between patient and clinician PS scores, with adverse PS changes reported approximately 15 months earlier by patients than by clinicians. ${ }^{6}$ Clearly, these observations support the general trend toward patient-reported rather than clinician-scored measures. Moreover, it is regrettable that although PS is typically scored at baseline as well as at every subsequent trial visit, its evolution over time (and per arm) is only rarely 
presented, although it may influence dropout rates. This information could shed some light on the clinical effect of a certain treatment, as was initially intended, as well as validate the baseline PS assessment.

Third, although poor PS scores (ECOG PS $\geq 3$ ) are predictors of overall survival and treatment tolerance, the prognostic value of good PS is very limited. Still, $39.5 \%$ of trials in the analysis by Abi Jaoude et al ${ }^{1}$ limit inclusion to patients with excellent PS (ie, ECOG $\leq 1$ ). Based on the current evidence, such a strict eligibility criterion is pointless and could easily be broadened to patients with reduced PS (eg, ECOG 2). ${ }^{7}$ As cancer treatments become less toxic, for example with targeted or immune-modulatory drugs, minimally invasive surgery, and modern volume-based radiation therapy, patient inclusion could foreseeably be broadened further. For instance, a retrospective study of 16,233 patients with solid tumors receiving contemporary chemotherapy regimens found negligible differences in relative dose intensity between patients with an ECOG PS of 0 versus $1-3 .^{8}$ Clearly, this issue is also related to the line of therapy, which is not always given enough weight. For instance, a patient with breast cancer with an ECOG PS of 4 at initial diagnosis may derive dramatic benefits from a clinical trial or indeed any systemic treatment, whereas a similar patient at the fifth line of therapy might be expected to benefit substantially less.

Still, replacing PS with an alternative that is straightforward but more objective is not easy. More robust (but also much more complex) validated tools exist for assessing functioning in elderly patients (eg, the comprehensive geriatric assessment) and in specific cancer types (eg, the hematopoietic cell transplantation-specific comorbidity index), as well as several predictive and prognostic scores for central nervous system malignancies. An assessment tool that can objectively evaluate overall functioning over several time points; is easily applicable for all ages, sexes, and cultures; and is (ideally) automated is critically needed. In that respect, the rapid expansion of consumer-grade digital mobile devices with integrated multisensory systems may offer a unique opportunity, for instance to objectify and automate the "up and about more than $50 \%$ of waking hours" from the ECOG PS scale. In fact, the facilitation of such "digital phenotyping" in oncology is an FDA priority. ${ }^{9}$ Emerging technologies may even allow blood biomarker panels for frailty to be identified in the future. ${ }^{10}$

\section{Recommendations}

We fully support Abi Jaoude et al $^{1}$ in their plea to include a wider target population in future cancer trials to ensure generalizability of results, while maintaining patient safety by careful monitoring. A possible solution could be to give patients with cancer a "right to participate" in clinical trials, obviously allowing some well-defined restrictions necessitated by the treatment under consideration (eg, liver function limitations in case of certain drugs) but no broad PS or age inclusion criteria. Additionally, regulatory organizations could mandate that subsequent approval depends on the applicability of the trial results for real-life situations (although this should not be permitted to delay the approval of effective new therapies). Research funders should also be encouraged to critically review all trials to ensure that recruitment is as broad as possible to reflect the real-world target population. Finally, registries could be set up for monitoring outcomes of new treatments to verify the "real-life effect" of clinical trial results, with special attention to those patients who were poorly represented in the original trials.

Disclosures: The authors have disclosed that they have no financial interests, arrangement affiliations, or commercial interests with the manufacturers of any products discussed in this article or their competitors.

Correspondence: Philip Poortmans, MD, PhD, Oosterveldlaan 24, 2610 Wilrijk, Belgium.

Email: Philip.poortmans@telenet.be

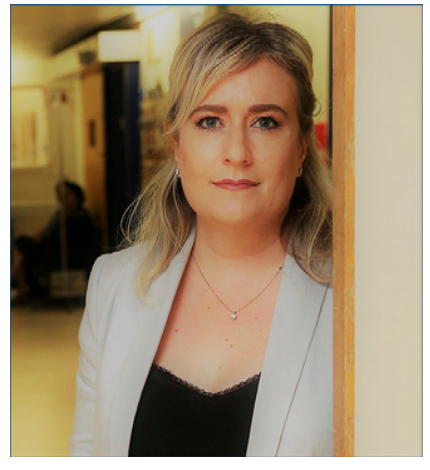

SHANI PALUCH-SHIMON, MBBS, MSc

Shani Paluch-Shimon, MBBS, MSc, is Director of Breast Oncology, Hadassah University Hospital, Jerusalem, Israel. She is Breast Cancer Subject Editor, Clinical Practice Guidelines, European Society of Medical Oncology (ESMO). 


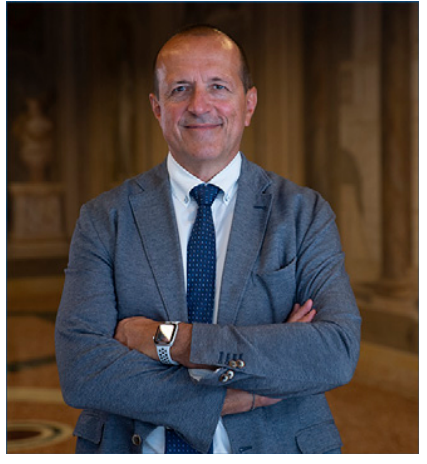

PHILIP POORTMANS, MD, PhD

Philip Poortmans, MD, PhD, radiation oncologist, is from the Department of Radiation Oncology, Iridium Network, and the University of Antwerp, Faculty of Medicine and Health Sciences, Wilrijk (Antwerp), Belgium. His main interests are breast cancer, quality assurance in clinical trials, malignant lymphoma, and rare tumors, and he has published primarily on these topics. $\mathrm{He}$ is former president of the ESTRO and ECCO and serves on several editorial boards, including as specialty editor for The Breast.

\section{References}

1. Abi Jaoude J, Kouzy R, Lin TA, et al. Performance status restriction in phase III cancer clinical trials. J Natl Compr Canc Netw 2020;18:1322-1326.

2. Murthy $\mathrm{VH}$, Krumholz HM, Gross CP. Participation in cancer clinical trials: race-, sex-, and age-based disparities. JAMA 2004;291:2720-2726.

3. Freedman RA, Foster JC, Seisler DK, et al. Accrual of older patients with breast cancer to alliance systemic therapy trials over time: Protocol A151527. J Clin Oncol 2017;35:421-431.

4. Rothwell PM. External validity of randomised controlled trials: "to whom do the results of this trial apply?" Lancet 2005;365:82-93.

5. Santacatterina M, Bottai M. Inferences and conjectures in clinical trials: a systematic review of generalizability of study findings. J Intern Med 2016;279:123-126.

6. Chow R, Bruera E, Temel JS, et al. Inter-rater reliability in performance status assessment among healthcare professionals: an updated systematic review and meta-analysis. Support Care Cancer 2020; 28:2071-2078.

7. Cheng S, Qureshi M, Pullenayegum E, et al. Do patients with reduced or excellent performance status derive the same clinical benefit from novel systemic cancer therapies? A systematic review and metaanalysis. ESMO Open 2017;2:e000225.

8. Denduluri N, Pratt DA, Wang Y, et al. Dose delays, dose reductions, and relative dose intensity in patients with cancer who received adjuvant or neoadjuvant chemotherapy in community oncology practices. J Natl Compr Canc Netw 2015;13:1383-1393.

9. US Food \& Drug Administration. FDA-ASCO public workshop: 2019 clinical outcome assessments in cancer clinical trials fourth annual workshzCop. Accessed September 4, 2020. Available at: https:// www.fda.gov/drugs/news-events-human-drugs/fda-asco-public-workshop-2019-clinical-outcomeassessments-cancer-clinical-trials

10. Rattray NJW, Trivedi DK, Xu Y, et al. Metabolic dysregulation in vitamin E and carnitine shuttle energy mechanisms associate with human frailty. Nat Commun 2019;10:5027.

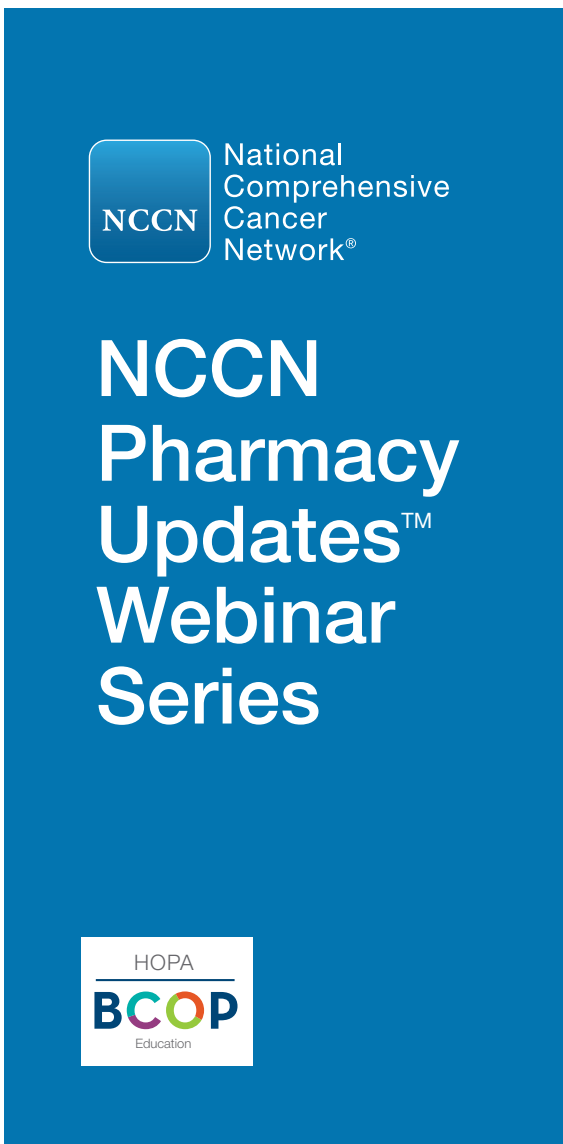

\section{Establishing a Place in Therapy for Biosimilars in Oncology} Wednesday, October 28, 2020 | 12:30 - 1:30 PM EDT

Rebecca J. Nelson, PharmD, BCOP Moffitt Cancer Center

\section{Treatment Advances for Pediatric B-Cell Non-Hodgkin's Lymphomas}

Tuesday, November 17, 2020 | 4:00 - 5:00 PM EST

Diana Wu, PharmD, BCPS, BCOP

St. Jude Children's Research Hospital

\section{education.nccn.org/pharmacy-updates}

NCCN designates each of these application-based activities for 1.0 contact hour ( 0.10 CEUs) of continuing education. Complete accreditation information is available online.

This activity is supported by an educational grant from AstraZeneca; Celgene Corporation; Coherus Biosciences; Novartis; and Servier Pharmaceuticals, LLC. This activity is supported by an independent educational grant from AbbVie. This activity is supported by an independent medical education grant from Advancer Accelerator Applications, a Novartis Company; and Mylan Inc. This educational activity is supported by a medical education grant from Exelixis, Inc. 